\title{
Elliptic flow studies in heavy-ion collisions using the CMS detector at the LHC
}

\author{
Vladimir Korotkikh for the CMS Collaboration ${ }^{* \dagger}$ \\ Skobeltsyn Institute of Nuclear Physics, Moscow State University, Leninskiye Gory, 119991 \\ Moscow, Russia \\ E-mail: Vladimir.Korotkikh@cern.ch
}

\begin{abstract}
The azimuthal anisotropy of charged particle emission in heavy ion collisions is a sensitive probe of the properties and the dynamical evolution of the produced matter. The systematic study of elliptic flow $\left(v_{2}\right)$, and higher order harmonics can yield information about the equilibration timescale, the nuclear equation of state and the viscosity in the different stages of the system evolution, as well as the initial state conditions of the heavy ion collisions. Experimentally, it is important to be able to reconstruct the flow using different methods, since they have different sensitivity to nonflow correlations and fluctuations which affect the extracted signal. In CMS, the nuclear reaction plane can be determined independently using several different detector subsystems.
\end{abstract}

The XIXth International Workshop on High Energy Physics and Quantum Field Theory, QFTHEP2010 September 08-15, 2010

Golitsyno, Moscow, Russia

\footnotetext{
*Speaker.

${ }^{\dagger}$ A footnote may follow.
} 


\section{Introduction}

The main goal pursued by the physics of heavy-ion processes is to study matter produced in collisions of relativistic nuclei, which is characterized by a high energy density, and to obtain its description on the basis of fundamental strong interaction theory (QCD).

At the present time, particle production in heavy-ion collisions and the dependence of particle distributions on the properties of a new dense medium are studied experimentally as functions of many variables. These include both kinematical variables (energy in the c.m. frame and particle transverse momentum and rapidity) and variables that are peculiar to nucleus-nucleus collisions and control the dimensions and shape of the collision region (impact parameter and the number of nucleons involved in the interaction process). According to data from the STAR [1], BRAHMS [2], PHENIX [3], and PHOBOS [4] experiments the energy density in the matter produced is ấij $5 \mathrm{GeV} / \mathrm{fm}^{3}$ [2]. This is higher than the value of about $1 \mathrm{GeV} / \mathrm{fm}^{3}$ expected on the basis of QCD lattice calculations $[5,6]$ for the critical energy density characteristic of the transition to a quarkgluon plasma (QGP). This condition is necessary, but it is not sufficient; fulfillment of at least three conditions is necessary for proving QGP formation - a high energy density, the thermalization of product matter, and deconfinement.

The collective effects manifestations, associated with new matter produced, in experimental results are reviewed in a work [7]. They concern the anisotropic particle distributions, the decreasing of nuclear modification factor at high $p_{T}$ and the suppression the outside peak in two particle correlation depending on reaction plane angle. Other comparison theoretical results with experimental heavy-ion collision data are referred in theoretical review articles of d'Enterria [8] and Borghini and Wiedemann [9].

In non central collisions between two nuclei the beam direction and the impact parameter vector define a reaction plane for each event. Initially, the overlap region of nuclei in the case of a nonzero impact parameter has an ellipsoidal shape in the transverse plane, the major axis of the ellipsoid being orthogonal to the reaction plane. If the thermalization of the matter produced in the overlap region occurs, it follows from the spatial anisotropy of the system that pressure gradients arise in this region: the pressure is maximal along the minor axis of the ellipsoid (in the reaction plane) and is minimal along the major axis. As a result, the azimuthal momentum distribution of particles becomes anisotropic. The observed azimuthal anisotropy of particles has been called the anisotropy of the transverse flux. The elliptic flow parameter, $v_{2}$, is defined as the second harmonic coefficient in the Fourier expansion of the particle azimuthal distribution with respect to the reaction plane.

The azimuthal anisotropy of particles exists only in the case where the yield of particles measured in the final state depends not only on physical conditions realized locally at the point of their production but also on the global geometry of the event being considered. Within a local relativistic theory, this non local information may be a source of a collective effect requiring interaction between many degrees of freedom localized at different points of the collision region. An anisotropic flux is of course a strong manifestation of collective dynamics in heavy-ion collisions.

This report is dedicated to studying the capability of the CMS detector [10] at the LHC to measure elliptic flow using calorimetry and the tracking system. The high tracking efficienc y and low rate of fake tracks at CMS, together with a fine tracker granularity provide a precise 
measurement of global event characteristics. The first measurements of proton-proton collisions with the CMS detector at the LHC began in November 2009 [11]. The heavy-ion programme at the LHC began with lead-lead collisions at $\sqrt{s}_{N N}=2.76 \mathrm{TeV}$ in November 2010. The first elliptic flow in $\mathrm{Pb}-\mathrm{Pb}$ collisions was measured by the ALICE co llaboration [12]. The $p_{T}$-dependence of $v_{2}\left(p_{T}\right)$ is similar to RHIC data.. Compared to RHIC $A u$ - $A u$ collisions at $\sqrt{s}_{N N}=200 \mathrm{GeV}$ the value increased by about $30 \%$ at medium centrality.

A measurement of the azimuthal anisotropy of particle production with respect to the reaction plane is an important tool for studying the properties of the dense matter created in ultra-relativistic heavy-ion collisions $[13,14]$. Ellipt ic flow in heavy-ion collisions at the LHC energy has been predicted to decrease, increase or sa turate compared to results at RHIC energies [15, 16, 17].

\section{The CMS detector}

The central feature of the CMS apparatus [10] is a $3.8 \mathrm{~T}$ superconducting solenoid of $6 \mathrm{~m}$ internal diameter. Located within the field volume are a silicon pixel and strip tracker, crystal electromagnetic calorimeter (ECAL) and brass scintillator hadron calorimeter (HCAL). Muons are measured in gas chambers embedded in the iron return yoke. CMS has extensive calorimeter coverage of the forward region with its HF $(3<|\eta|<5.2)$, CASTOR $(5.3<\eta<6.6)$ and Zero Degree $(|\eta|>8.3)$ calorimeters.

Near mid rapidity $(|\eta|<2.4)$ charged particles are tracked by three layers of silicon pixel detectors, made of 66 million pixels with dimensions $100 \times 150 \mu \mathrm{m}^{2}$, followed by ten microstrip layers, with strips of pitch between 80 and $180 \mu \mathrm{m}$. The silicon tracker allows determination of the vertex position with $\sim 15 \mu \mathrm{m}$ accuracy. The good momentum resolution of the tracker allows us to clearly resolve the $\Upsilon$-family. The calorimeter cells are grouped in projective towers, of granularity $\Delta \eta \times \Delta \phi=0.087 \times 0.087$ at central rapidities and $0.175 \times 0.175$ at forward rapidities. The ECAL has an energy resolution of better than $0.5 \%$. The HCAL combined with the ECAL measures jets with an energy resolution $\Delta E / E \approx 100 \% / \sqrt{E} \oplus 5 \%$. Muons are measured in the pseudorapidity window $|\eta|<2.4$, with three technologies: drift tubes, cathode strip chambers, and resistive plate chambers. Matching the muons to the tracks measured in the tracker results in a transverse momentum resolution of $1-5 \%$ up to $1 \mathrm{TeV} / c$.

\section{Analysis}

There exist a wealth of anisotropic flow measurement methods, each of which has its advantages and limitations. Here we have used a $v_{2}$ determination method based on the event plane angle measurement. Usually the true elliptic flow coefficient in the event plane (EP) method is evaluated by dividing the observed $v_{2}$ value by a $R$ factor, [18], which accounts for the event plane resolution:

$$
v_{2}\{E P\}=\frac{\nu_{2}^{\mathrm{obs}}\{E P\}}{R}=\frac{\left\langle\cos 2\left(\varphi-\Psi_{2}\right)\right\rangle}{\left\langle\cos 2\left(\Psi_{2}-\Psi_{R}\right)\right\rangle} .
$$

Here the event plane angle $\Psi_{2}$ is the estimate of the true reaction plane angle $\Psi_{R}$. The mean was taken over all charged particles in a given event and then over all events. In order to avoid the trivial autocorrelation of particles, the event plane angle $\Psi_{2}$, and hence $<R$, are calculated from 
the angular distribution of a sample of events, and $v_{2}$ from another event sample with the same multiplicity. The samples can be selected, for instance, in two distinct regions of pseudorapidity, such as $\eta<0$ and $\eta>0$.

Other techniques like the cumulant [20] and Lee-Yang zeros [21] methods will also be used for elliptic flow measurement with the CMS detector. In reference [22], it was shown that the application of the Lee-Yang zeros method makes it possible to suppress the contribution of nonflow effects and to measure the parameter $v_{2}\left(p_{T}\right)$, to a good precision of about 10 to $20 \%$ under the conditions of forthcoming experiments with the CMS detector.
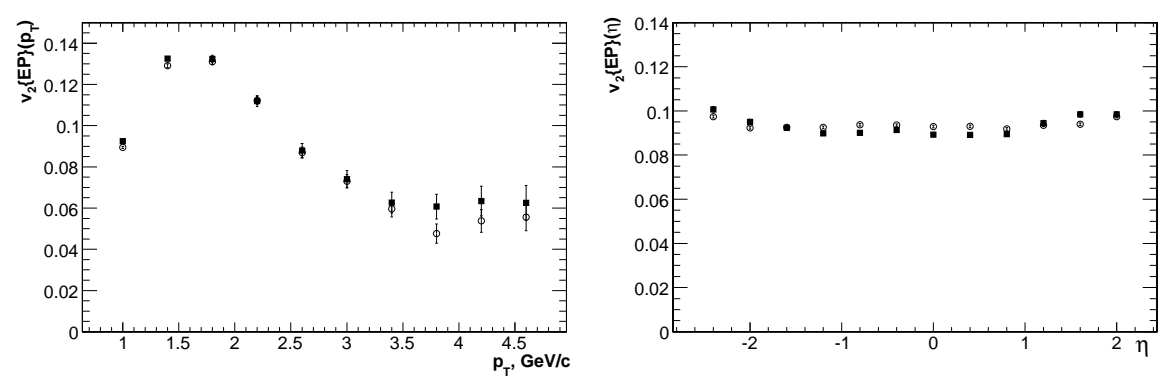

Figure 1: The $p_{T}$ dependence and $\eta$ dependence of $v_{2}\{E P\}$ in $P b-P b$ collisions for impact parameter $b=9$ $\mathrm{fm}$, calculated with the simulated (open circles) and reconstructed events (closed squares). Statistical errors are shown for $10^{5}$ events. Nonflow systematic uncertainties not included.

For the estimation of the azimuthal anisotropy of particles in heavy-ion collisions, the HYDJET event generator [23] was used with full GEANT simulation of the CMS detector responses.

Study of event plane resolution with CMS calorimeters. It was found that the CMS calorimetric system can be used for the determination of the event plane, using the energy responses of the calorimeter towers [10]. Although the anisotropic flow is maximal at midrapidity, the much larger total energy deposition in the CMS calorimeter endcaps $(1.5<|\eta|<3$.) results in reduced relative fluctuations and, accordingly, in a much better event plane resolution. Moreover, energy flow measurements in the endcaps are less sensitive to the magnetic field than in the barrel region $(|\eta|<1.5)$.

Study of $v_{2}$ reconstruction with the CMS tracker. A sample of $10^{5} \mathrm{~Pb}-\mathrm{Pb}$ events at impact parameter $b=9 \mathrm{fm}$ at $\sqrt{s}=5.5 \mathrm{TeV}$ per nucleon pair within the pseudorapidity window $|\eta|<2.4$ (the CMS tracker acceptance) was generated. The standard settings were used to reconstruct tracks (i.e. more than 12 hits per track and a track fit probability above $1 \%$ ). A cut on $p_{T}>0.9 \mathrm{GeV} / c$ was set in both simulated and reconstructed events. The number of reconstructed tracks per event is about 170 at this centrality.

The differential $p_{T}$ and $\eta$ dependencies of the elliptic flow in $P b-P b$ collisions for impact parameter $b=9 \mathrm{fm}$ are shown in Fig. 1, respectively. For the $p_{T}$ dependence, two sub-event sets were used, with $\eta>0$ and $\eta<0$. For the $\eta$ dependence, the factor $R$ in each histogram bin was calculated using particles from other bins (excluding neighboring bins).

The CMS detector at the LHC is able to determine the reaction plane using the calorimeters and the tracker. The transverse momentum dependence of the elliptic flow coefficient $v_{2}$ can be reconstructed in the CMS tracker with high accuracy. 


\section{Study of other collective phenomena}

Viscosity and quenching The well-known nuclear modification factor has a strong and $p_{T^{-}}$ independent suppression. For $A u$ - $A u$ collisions of $010 \%$ centrality a suppression of single-particle inclusive spectra by a factor of about five in the region $p_{T}>2 \mathrm{GeV} / \mathrm{c}$ is observed at intermediate rapidities. The factor $R_{A A}$ becomes greater in the reaction plane than in the direction orthogonal to this plane. This phenomena is interpreted as a quenching of parton in medium. See for example [24] and references therein. Some quenching models provide a good description of the reaction plane dependence of $R_{A A}$. In the same region the parameter $v_{2}\left(p_{T}\right)$ has a bend and $v_{2}\left(p_{T}\right)$ begins to decrease. This decre ase may be in principle explained by the quenching [23, 24] and also by a small viscosity of the medium [25]. The quenching model alone cannot describe both phenomena-it underestimat es the values of $v_{2}\left(p_{T}\right)$ [24]. Viscous hydrodynamic models do not predict the factor $R_{A A}$.

Ridge effect and collective elliptic flow The Ridge effect is observed in $A u$ - $A u$ collisions [26] as a long range pseudorapidity correlation. The latest measurements from STAR [27] and PHENIX [28] were carried out with respect to the reaction plane. They show that the Ridge effect drops if the azimuth of the trigger particle is perpendicular to the reaction plane and the elliptic flow is present. A combined study of two effects is thus necessary.

Away-side peak suppression in the direction perpendicular to reaction plane In two particle azimuthal correlations the away-side peak is not manifested in central $A u-A u<$ collisions, but it is observed in peripheral ones. The absence of correlations at $\delta \varphi=\pi$ in central $A u-A u$ collisions is indicative of the suppression of hard scattered partons or their fragments in a dense product medium. In the directions opposite to the reaction plane the effect of away-side peak suppression is much stronger [28]. This corresponds to the pattern where, in two jet events, one jet originates from a parton that did not undergo interaction in traversing the medium, while the other originates from a parton that passed through a layer of matter of this medium. If, in this case, the first parton es caped from the surface of the ellipsoidal region of the medium in the direction orthogonal to the reaction plane, the second parton in the two jet event flies in the opposite direction and traverses a thicker layer of matter, losing a greater amount of energy and not forming a hadron jet peak.

These different but coupled phenomena (at least in the same kinematic region) may have the same underlying physical cause, which should be investigated by CMS at the LHC.

\section{Acknowledgments}

I would like to thank the members of the CMS collaboration for providing the materials. I wish to express the gratitude to the organizers of the XIXth International Workshop High Energy Physics and Quantum Field Theory for the opportunity to give my talk.

\section{References}

[1] J. Adams et al. (STAR), Nucl. Phys. A757 (2005) 102.

[2] I. Arsene et al. (BRAHMS), Nucl. Phys. A757 (2005) 1.

[3] .K. Adcox et al. (PHENIX), Nucl. Phys.A757 (2005) 184. 
[4] B. Back et al. (PHOBOS), Nucl. Phys. A757 (2005) 28.

[5] F.Karsch, Nucl. Phys. A698, 199 (2002).

[6] Z. Fodor, in proceedings of 4th International Workshop on Critical Point and Onset Deconfinement, POS CPOD07:027,2007 [arXiv:0712.2930]

[7] V. L. Korotkikh et al., Phys.Atom.Nucl. 72 (2009) 1951.

[8] D. d'Enterria, J. Phys. G34 (2007) S53.

[9] N. S .Borghini and U. A. Wiedemann, J. Phys. G: Nucl.Part.Phys. G35 (2008) 023001.

[10] G. L. Bayatian et al. (CMS Collaboration), CMS technical design report, volume II: Physics performance, J. Phys. G34 (2007) 995.

[11] V. Khachatryan et al. (CMS Collaboration) J. High Energy Phys. 1002 (2010) 041.

[12] K. Aamodt Elliptic flow of charged particles in Pb-Pb collisions at 2.76 TeV et al. (ALICE) [arXiv:1011.3914]

[13] J. Y.Ollitrault, Phys.Rev. D46 (1992) 229.

[14] H. Sorge, Phys. Rev.Lett.D82 (1999) 2048.

[15] N. Armesto, Predictions for the heavy-ion programme at the Large Hadron Collider. [arXiv:0903.1330].

[16] G. Kh. Eyyubova et al., Phys.Rev. C80 (2009) 064907.

[17] E. Zabrodin et al..,J. Phys. G37(2010) 094060.

[18] A.M.Poskanzer, S.A.Voloshin, Phys.Rev. C58 (1998) 1671.

[19] S.A.Voloshin, Y.Zhang, Z.Phys. C70 (1996) 665, .

[20] N.Borghini, P.M.Dinh, J.-Y.Ollitrault, Phys.Rev. C64 (2001) 054901.

[21] R.S.Bhalerao, N.Borghini, J.-Y.Ollitrault, Nucl.Phys A727(2003) 373 .

[22] V.L.Korotkikh et al.,, Phys.Atom.Nucl. 71 (2008) 2142.

[23] I.P.Lokhtin, A.M.Snigirev, Eur.Phys.J. C46 46, (2006) 211.

[24] A. Adare et al.. (PHENIX), Phys. Rev. Lett. 105 (2010) 14230.

[25] M. Luzum and P. Romatschke, Phys. Rev. C78 (2008) 034915.

[26] B. Abelev et al.. (STAR), Phys. Rev. C80 (2009) 064912.

[27] H. Agakishiev et al.. (STAR). Measurements of Dihadron Correlations Relative to the Event Plane in $\mathrm{Au}+\mathrm{Au}$ Collisions at $\sqrt{s_{N N}}=200 \mathrm{GeV}$ [arXiv: 1010 .0690].

[28] A. Adare et al.. (PHENIX), Suppression of away-side jet fragments with respect to the reaction plane in $\mathrm{Au}+\mathrm{Au}$ collisions at $\sqrt{\left(s_{N} N\right)}=200 \mathrm{GeV}$ [arXiv:1010.1521]. 